\title{
Unique N-terminal sequences in two Runx1 isoforms are dispensable for Runx1 function

Sebastian Nieke ${ }^{1,2}$, Nighat Yasmin ${ }^{1,5}$, Kiyokazu Kakugawa $^{3}$, Tomomasa Yokomizo ${ }^{4,6}$, Sawako Muroi ${ }^{1}$ and Ichiro Taniuchi ${ }^{1^{*}}$ (D)

\begin{abstract}
Background: The Runt-related transcription factors (Runx) are a family of evolutionarily conserved transcriptional regulators that play multiple roles in the developmental control of various cell types. Among the three mammalian Runx proteins, Runx1 is essential for definitive hematopoiesis and its dysfunction leads to human leukemogenesis. There are two promoters, distal (P1) and proximal (P2), in the Runx1 gene, which produce two Runx 1 isoforms with distinct N-terminal amino acid sequences, P1-Runx1 and P2-Runx1. However, it remains unclear whether P2-Runx specific N-terminal sequence have any specific function for Runx1 protein.

Results: To address the function of the P2-Runx1 isoform, we established novel mutant mouse models in which the translational initiation AUG (+1) codon for P2-Runx1 isoform was modulated. We found that a truncated P2-Runx1 isoform is translated from a downstream non-canonical AUG codon. Importantly, the truncated P2-Runx1 isoform is sufficient to support primary hematopoiesis, even in the absence of the P1-Runx1 isoform. Furthermore, the truncated P2-Runx1 isoform was able to restore defect in basophil development caused by loss of the P1-Runx1 isoform. The truncated P2-Runx1 isoform was more stable than the canonical P2-Runx1 isoform.
\end{abstract}

Conclusions: Our results demonstrate that the N-terminal sequences specific for P2-Runx1 are dispensable for Runx1 function, and likely serve as a de-stabilization module to regulate Runx1 production.

Keywords: Runx1 proteins, Promoters, Isoform, Hematopoiesis, Translational start site

\section{Background}

Runx transcription factor complexes are evolutionarily conserved heterodimers that consist of an $\alpha$-subunit, referred to as the Runx protein, and a non-DNA binding $\beta$-subunit. Runx complexes recognize a specific DNA sequence (5'-PyGPyGGT-3') through their conserved Runt-domain [1-3]. In mammals, three genes encode three distinct Runx proteins, Runx1, Runx2 and Runx3. Ablation or attenuation of each Runx gene function in several species revealed that Runx complexes play pivotal roles in the development of many cell types $[2,4,5]$. For example, Runx1 is required for definitive hematopoiesis in vertebrates. Genetic ablation of Runx1

\footnotetext{
* Correspondence: ichiro.taniuchi@riken.jp

${ }^{1}$ Laboratory for Transcriptional Regulation, RIKEN Center for Integrative Medical Sciences (IMS). 1-7-22 Suehiro-cho, Tsurumi-ku, Yokohama 230-0045, Japan

Full list of author information is available at the end of the article
}

in mice blocks hematopoietic stem cell (HSC) generation and results in embryonic lethality at approximately 12.5 days post-coitum $(\mathrm{dpc})$ and hemorrhages in the central nervous system (CNS) [6, 7]. Runx1 has also been implicated in human leukemia [8]. Generation of fusion proteins such as RUNX1/ETO and RUNX1/Evi1 through leukemic associated-chromosomal translocation are frequently observed in acute myeloid leukemia (AML) [9]. In addition, mutations in the RUNX1 gene have been observed in a significant fraction of AML patients [10]. Thus, understanding of how Runx1 expression and function are regulated is fundamental to the field of hematology.

All mammalian Runx genes are transcribed from distal (P1) and proximal (P2) promoters [3]. The P1-Runx1 promoter is located $130 \mathrm{~kb}$ upstream of the P2-RunxI promoter in the murine Runx1 locus [11]. The 5' untranslated regions (UTR) is short in the P1-Runx1 transcript, whereas in $P 2-R u n x 1$ transcript, it spans more than $1.6 \mathrm{~kb}$ and contains GC-rich regions [11] and 
a putative internal ribosomal re-entry (IRES) element upstream of the translation initiation AUG $(+1)$ [12]. Thus, atypical cap-independent and IRES-dependent translation mechanisms are involved in the translation of P2-Runx1 transcripts. In addition to these differences in translational control, the expression patterns of $P 1$ and P2-Runx1 transcripts are also different [13-15]. Expression of the P2-Runx 1 transcript is detected earlier in mouse embryogenesis than the P1-Runx1 transcript $[13,16,17]$. In addition, the products of $P 1$ - and $P 2$ Runx1 transcripts, hereafter referred to as P1-Runx1 and $\mathrm{P} 2$-Runx1 proteins, have distinct $\mathrm{N}$-terminal sequences $[3,13]$; ASDSIFESFPSYPQCFMR and RIPV are unique within the N-terminus of P1-Runx1 and P2-Runx1 proteins, respectively. These isoforms were previously shown to be different in terms of their effect of exogenous expression on cell growth and differentiation of myeloid progenitor cell line [13]. This suggests that distinct $\mathrm{N}$-terminal sequences could confer different functionality to the P1-and P2-Runx1 isoform. The physiological roles of P1-Runx1 and P2-Runx1 proteins were examined in animal models. In contrast to the embryonic lethality caused by a total loss of Runx1 function (Runx $1^{\Delta / \Delta}$ mice), mice lacking only the P1-Runx1 isoform were alive at birth $[16,17]$. These mice did not display any apparent defects in early hematopoiesis in the fetal liver. A lack of P1-Runx1 did, however, result in impaired colony forming activity in hematopoietic progenitors in the yolk-sac of $11.5 \mathrm{dpc}$ embryos [16] and affected differentiation of lymphoid tissue inducer (LTi) cells [18]. In adult Runxi $1^{P I N / P 1 N}$ mice, basophil development was also impaired [19]. On the other hand, a mouse model with substantially reduced levels of the P2-Runx1 transcript was also generated by insertion of the neomycin resistant gene $\left(\right.$ neo $\left.^{r}\right)$ upstream of $P 2$ Runx1 promoter [20]. Mice homozygous for this

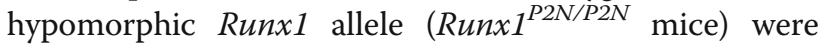
born alive but died a few days after birth [20]. Colony forming activity in embryonic hematopoietic progenitors was more severely impaired in $R u n x 1^{P 2 N / P 2 N}$ than in Run $x 1^{P 1 N / P 1 N}$ embryos [16]. Furthermore, when compound mutations were generated between the Runx1 null mutation $\left(\operatorname{Run} x 1^{\Delta}\right)$ and either the $\operatorname{Runx} 1^{P I N}$ or $\operatorname{Run} x 1^{P 2 N}$, embryonic lethality and liver anemia were observed in $\operatorname{Run} x 1^{P 2 N / \Delta}$ embryos, but not in $\operatorname{Run} x 1^{P 1 N / \Delta}$ embryos [16]. These observations indicated that the P2Runx1 isoform is more crucial for definitive hematopoiesis than the P1-Runx1 isoform. Activation of P2-Runx1 promoter activity generally occurs earlier than that of the P1-Runx1 promoter [13,17, 20,21], and the P1-Runx1 promoter, which has conserved functional tandem Runx recognition sites [13, 22], appeared later during evolution presumably in ancestral vertebrates [3]. Therefore, the predominant requirement of P2-Runx1 for definitive hematopoiesis is thought to reflect its role in activating the P1-Runx1 promoter for promoter switching. However, it remains unclear whether P2Runx1 specific $\mathrm{N}$-terminal sequences, RIPV, are required for early hematopoiesis or not.

In the current study, we generated novel Runx1 mutant alleles by replacing the translational start AUG $(+1)$ codon of the P2-Runx1 isoform with different STOP codons (UAG or UAA). These models gave unexpected results, showing that the expression of a truncated Runx1 isoform from the P2-Runx1 promoter alone is sufficient for early hematopoiesis.

\section{Methods}

\section{Construction of the target vectors and generation of chimera mice}

A phage clone containing genomic regions surrounding the P2-Runx1 promoter was isolated from a phage library purchased from STRATAGEN. A 3' side short arm fragment was amplified by PCR and was ligated into the pL2Neo2 vector after sequencing, generating a pBlueNeoSA1 vector. A fragment containing the loxPflanked neo $o^{r}$ gene and the 3' short arm was prepared from the pBlueNeoSA1 vector by XbaI/ClaI digestion and was cloned into the $\mathrm{pBS}-\mathrm{TK} 1$ vector, generating a pR1pNSATK vector. A $1.9 \mathrm{~kb}$ XhoI-SpeI fragment corresponding to chr16:92,695,251-92,697,182 (mm9) was amplified by PCR and cloned into the pBluescript vector, generating a pR1pEXII vector. A 7.4 kb $5^{\prime}$ long arm fragment corresponding to chr16:92,697,759-92,705,187 was prepared from the phage clone by NotI and SmaI digestion and was cloned into the pBluescript, generating a pR1pLA1 vector. A $1.9 \mathrm{~kb}$ fragment cut out from the pR1pEXII vector by HincII/KpnI digestion was ligated into the SmaI/KpnI cleaved pR1PLA1 vector, generating a pR1PLAEXWT vector, in which a 580 bp SmaI-XhoI region corresponding to chr16:92,697,182-92,697,761 was missing from the $5^{\prime}$ long arm. A fragment containing the TAG replacement mutation was created by overlap PCR and ligated into the SmaI/SpeI-cleaved pR1pLAEXWT vector, generating a pR1pLAEXMu vector. Finally, a $9.3 \mathrm{~kb}$ NotI-SpeI fragment prepared from the pR1pLAEXMu vector was ligated into the NotI/ XbaI-cleaved pR1pNSATK vector.

To generate the target vector for the Runx $1^{\text {P2TAA }} \mathrm{mu}$ tation, overlap PCR was performed with appropriate primers. After sequencing, the $0.8 \mathrm{~kb}$ PCR product harboring SmaI and KpnI at the $5^{\prime}$ and $3^{\prime}$ ends, respectively, and the $7.4 \mathrm{~kb}$ NotI-SmaI fragment prepared from the pR1pLA1 vector, were ligated using a trimolecular reaction into the NotI/KpnI-cleaved pBluescript vector, generating a pRIpSmaI vector.

These target vectors $(30 \mu \mathrm{g})$ were linearized by ClaI digestion before transfection into the ES cell line, M1, by 
electroporation using a GenePulserII (Bio-Rad). After selecting cells with $350 \mu \mathrm{g} / \mathrm{ml} \mathrm{G418} \mathrm{(GENETICIN,} \mathrm{Gibco)}$ and $2 \mu \mathrm{M}$ ganciclovir (Wako, 078-04481), individual colonies were subjected to PCR screening with appropriate primers to identify clones that had undergone homologous recombination. ES cell aggregation was performed by the animal facility group at RIKEN IMS.

\section{Mice}

This study was carried out in accordance with guidelines for animal care of the RIKEN Yokohama Campus. Animal experimental protocol was approved by Safety Department at RIKEN Yokohama Campus (Permit Number: 28-017(2)). All mice were maintained in the animal facility at the RIKEN IMS and all animal procedures were in accordance with the institutional guidelines for animal care and were approved by the safety section in RIKEN Yokohama Campus.

\section{Flow cytometry}

Single-cell suspensions were prepared from thymus, spleen, bone marrow and fetal liver, and were stained with the following antibodies, all purchased from BDBiosciences: CD4 (RM4-5), CD8 (53-6.7), CD49b (DX5), CD117/c-Kit (2B8), IgE (R35-72) and ScaI (E13-161.7). The antibody for FceRI (MAR-1) was from eBiosciences. Multi-color flow cytometry analysis was performed using a FACS CANTO II (BD-Biosciences), and data were analyzed using FlowJo software (Tree Star).

\section{Colony forming assay}

A colony forming assay on methylcellulose agar was conducted according to the manufacturer's instructions (Stem Cell Technologies). Single cell suspensions of total fetal liver cells from E11.5 dpc embryos were mixed with MethoCultTM GF M3434 containing SCF, IL3, IL6 and Epo, and plated onto $6-\mathrm{cm}$ dishes, according to the protocols provided. Colony numbers were counted after seven days of culture in a humidified $\mathrm{CO}_{2}$ incubator.

\section{Whole-mount immunostaining}

Whole-mount immunostaining of the embryos was performed as previously described [23]. Primary antibodies used were anti-c-Kit (2B8; BD Biosciences) and biotinylated anti-CD31 (MEC13.3; BD Biosciences). The secondary antibody and labeled streptavidin used were goat anti-rat IgG-Alexa Fluor 647 (Invitrogen) and Cy3-streptavidin (Jackson ImmunoResearch Laboratories). Immunostained embryos were mounted in a 1:2 mix of benzyl alcohol and benzyl benzoate (BABB) to increase tissue transparency and analyzed using a confocal microscope (Zeiss LSM 510 Meta, Plan-Neofluar $20 \times /$ NA 0.5). Three-dimensional projections were generated from $\mathrm{z}$-stacks using LSM Image Browser (Zeiss).

\section{Rt-Pcr}

Total RNA from embryos and cells was prepared by using TRIzol (ThermoFisher Scientific). After treatment with DNase, $1 \mu \mathrm{g}$ of total RNA was used to synthesize cDNA using SuperScript ${ }^{\mathrm{TM}}$ II Reverse Transcriptase (Invitrogen). Primers used to amplify P1- and P2-Runx2 transcripts were P1-Runx1-F:5' - CTTCAGGAGAGGTGCGTTTTC G -3', P2-Runx1-F: 5' - CCTCCGGTAGTAATAAAGGC TTC-3', and Runx1-R: 5' - ATGACGGTGACCAGAGTG CC $-3{ }^{\prime}$.. Primers for Mcpt8 and bactin were described previously [19].

\section{Immunoblotting}

Cells were lysed with lysis buffer (20 mM Tris, $150 \mathrm{mM}$ $\mathrm{NaCl}, 10 \mathrm{mM} \mathrm{MgCl} 2,0.1 \% \mathrm{NP}-40)$ containing protease inhibitors (Complete Mini, 11,836,153,001, Roche) and incubated on ice for $20 \mathrm{~min}$. The supernatant was collected after centrifugation at 10,000 rpm for $10 \mathrm{~min}$ at $4{ }^{\circ} \mathrm{C}$, mixed with Laemmli sample buffer (Bio-Rad), and subjected to $10 \%$ SDS- PAGE followed by transfer to a membrane. Samples were probed with an anit-Runx1 antibody [24] or an anti- $\beta$-actin antibody (Sigma, A3853). Immunocomplexes were detected using ECL reagents (Amersham).

\section{Proteasome inhibitor treatment}

One million of $\mathrm{CD}^{+}$spleen cells were cultured in RPMI1640 media containing10\% FBS with or without 10uM MG132 (Calbiochem, Cat\# 474791) for one hour before preparation of cell lysate.

\section{Results}

\section{Characterization of Runx $1^{\text {P2TAG/P2TAG }}$ mutant mice}

To examine the physiological function of the P2-Runx1 isoform, it is useful to establish a mouse model that specifically lacks this isoform. The extreme 3' sequences of exon II contains a splice acceptor signal for the P1-Runx1 transcript, and therefore all sequences after this splice acceptor are shared with the P1-Runx1 coding sequences (Fig. 1a). We therefore designed a target vector that would replace the translation start codon, AUG, with a stop codon, UAG, to eliminate translation of P2-Runx1 isoform while maintaining intact P1-Runx1 expression (Fig. 1b). We included an additional deletion to remove around 200 bp upstream of the putative transcriptional start site (TSS) of the P2-Runx1 transcript and part of the 5'UTR of the P2-Runx1 transcript (Fig. 1B). ES clones that underwent homologous recombination were screened first by PCR and verified by Southern blotting using a probe located at the 3 ' end (Fig. 1c). ES clones harboring the P2promoter deletion were confirmed by PCR (Fig. 1d), while incorporation of the TAG mutation was confirmed by sequencing (Fig. 1e). After removal of the neo ${ }^{r}$ gene in ES clones by transient transfection of a Cre expression vector, 


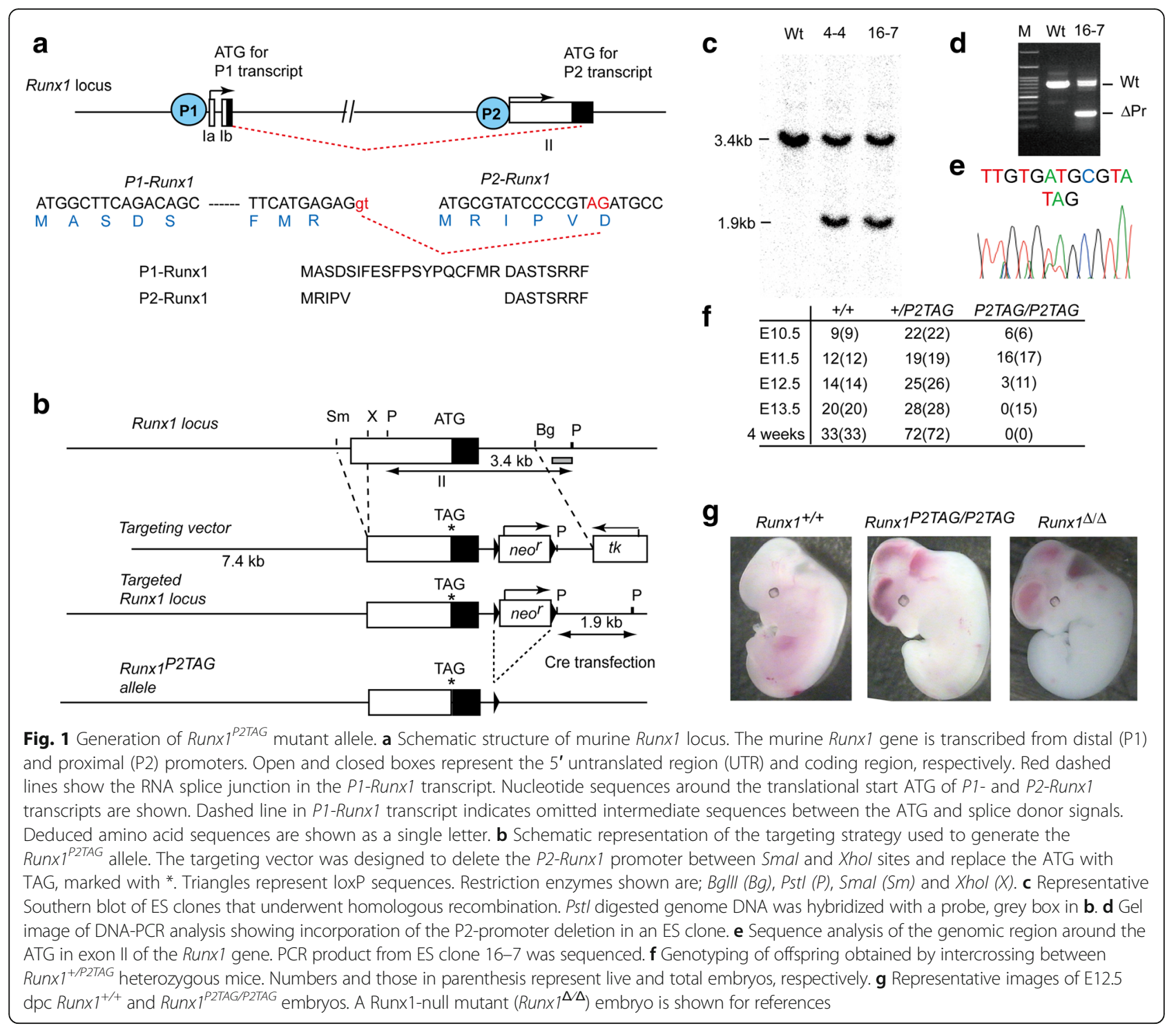

ES clones were aggregated with eight-cell embryos to generate chimeric mice. These mice were used to establish a mouse line harboring the $\operatorname{Run} x 1^{\text {P2TAG }}$ allele. Intercrossing heterozygous mice revealed no homozygous mice (Runx1 $1^{\text {P2TAG//P2TAG }}$ ) in the 4-week-old offspring (Fig. 1f). Analysis of embryos at different developmental stages revealed that Runx $1^{\text {P2TAG/P2TAG }}$ embryos were alive at $11.5 \mathrm{dpc}$, but dead at $13.5 \mathrm{dpc}$ (Fig. 1f). In addition, at $12.5 \mathrm{dpc}$, most of the Runx $1^{P 2-}$ TAG/P2TAG embryos exhibited hemorrhagic regions in the brain and spinal cord (Fig. 1g), as was also seen in Runx1-null mutant $\left(R u n x 1^{\Delta / \Delta}\right)$ embryos [7]. Thus, the Run $x 1^{\text {P2TAG }}$ mutation resulted in embryonic lethality around $12.5 \mathrm{dpc}$, with hemorrhagic characteristics similar to those observed in Runx1-null mutants. These findings led us to next examine definitive hematopoiesis in the RUnx $1^{\text {P2TAG/P2TAG }}$ embryos.
Flow cytometry analyses of $11.5 \mathrm{dpc}$ embryos showed a decrease in the percentage of lineage-marker-negative

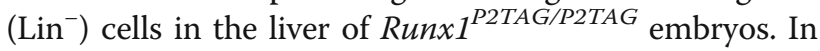

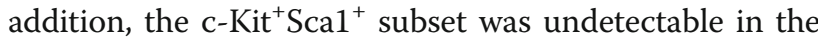
$\mathrm{Lin}^{-}$liver population of Runx1 P2TAG/P2TAG embryos (Fig. 2a), indicating that HSC generation was totally abrogated in these embryos. Consistent with these findings, no hematopoietic colonies arose on methylcellulose dishes comprising Runx1 $1^{\text {P2TAG/P2TAG }}$ fetal liver cells, while such colonies were seen in wild-type and heterozygous fetal liver cells (Fig. 2b). The lack of c-Kit expressing cells around CD31 expressing endothelial cells in Run $x 1^{\text {P2TAG/P2TAG1 }} 11.5 \mathrm{dpc}$ embryos was also confirmed by immunohistochemical staining (Fig. 2c). Consistent with lack of HSC, western blot using antibody that recognize both Runx1 and Runx 3 proteins [24] failed to detect these proteins in fetal liver cells of 


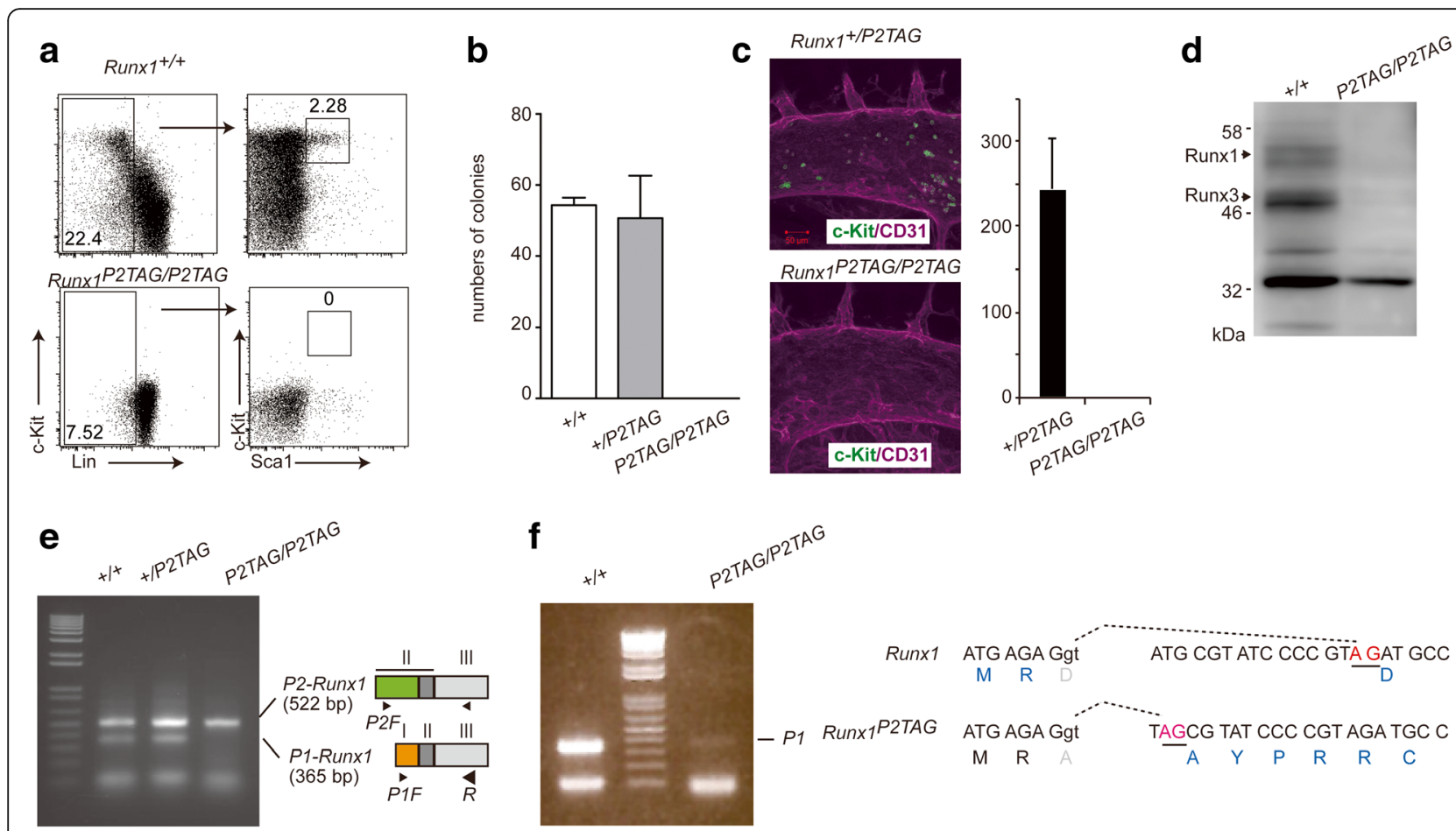

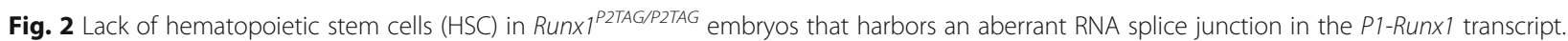
a Representative dot plots of fetal liver cells from Runx $1^{+/+}$and Runx $1^{\text {P2TAG/P2TAG }} 11.5 \mathrm{dpc}$ embryos. Lin-negative (Lin ${ }^{-}$) cells were analyzed for Scal and c-Kit expression. $\mathbf{b}$ Results of three independent colony forming assays of fetal liver cells. No colonies formed from Runx $1^{\text {P2TAG/P2TAG }}$ cells. c Immunohistochemical analysis of c-Kit and CD31 expression in the dorsal aorta of $11.5 \mathrm{dpc}$ embryos. In control Runx $1^{+/ P 2 T A G}$ samples, round c-Kit positive cells were seen budding from CD31 positive endothelial cells, whereas those cells were undetectable in Runx $1^{\text {P2TAG/P2TAG }}$ embryos. Right graph shows a summary of the numbers of c-Kit expressing cells in the dorsal aorta of three embryos. Mean \pm SD. $\mathbf{d}$ Immunoblot showing expression

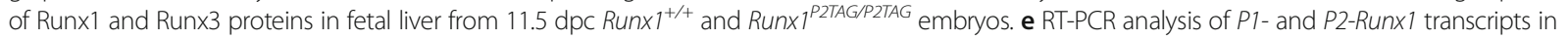

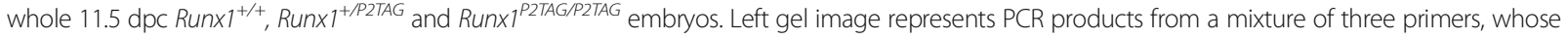
positions are illustrated on the right. I, II and III represent corresponding exons. f RT-PCR analysis focusing on the P1-Runx 1 transcript using two primers, P1F and R. PCR product corresponding to the P1-Runx 1 transcript is indicated. All eight P1-Runx 1 transcripts from the Runx $1^{\text {P2TAG/P2TAG }}$ embryos examined contained an aberrant splice junction between exon I and II, which resulted from upstream TAG sequences. AG in red font indicates a splice acceptor signal and dashed line indicates a splice junction. Aberrant splicing cause a frame shift and deduced amino acids are shown as a single letter

Runx $1^{\text {P2TAG/P2TAG }} 11.5 \mathrm{dpc}$ embryo (Fig 2d). We concluded from these observations that the Runxi $1^{\text {P2TAG }}$ allele behaves as functionally null allele.

Next, we examined the expression of P1- and P2Runx1 transcripts using RT-PCR with forward primers specific to each isoform and a common reverse primer (Fig. 2e). In this setting, both $P 1$ - and P2-Runx1 transcripts were detected in mRNA prepared from whole wild-type and heterozygous $11.5 \mathrm{dpc}$ embryos, but the amount of P2-Runx1 transcript was higher than P1Runx1. Of note, despite removal of the putative P2promoter region, the P2-Runx 1 transcript could still be detected in Runx $1^{\text {P2TAG/P2TAG }}$ embryos at similar levels to control embryos. On the contrary, the P1-Runx1 transcript was barely detectable in Runx $1^{\text {P2TAG/P2TAG }}$ embryos (Fig. 2f). Thus, we performed RT-PCR with two primers that specifically amplify it, the P1-Runx1 transcript was detected also in Runx $1^{\text {P2TAG/P2TAG }}$ embryos, albeit at very low levels compared to $R u n x 1^{+/+}$embryos
(Fig. 2f). In order to verify whether the PCR products amplified from Runx $1^{\text {P2TAG/P2TAG }}$ embryos was the real P1-Runx1 transcript, the PCR product was cloned into a plasmid and sequenced. While sequences at the $5^{\prime}$ end were the same as P1-Runx1, we found addition sequences in the middle of all eight clones sequenced. Sequence alignment revealed that the insertion of these additional sequences stemmed from aberrant usage of the inserted TAG as a novel splice acceptor signal (Fig. 2f). Importantly, this aberrant splicing resulted in a frame shift (Fig. 2e), that eventually created a stop codon upstream of the Runt-domain. Thus, the aberrant P1Runx 1 transcript transcribed from the Runx $1^{\text {P2TAG }}$ allele generates mostly short truncated peptides that consist of 74 amino acids and lacks the Runt-domain. Sequence analyses of P1-Runx1 transcripts expressed in 11.5dpc Run $x 1^{+/ P 2 T A G}$ fetal liver found 3 aberrant P1-Runx1 among 31 clones sequence, indicating that non-sense mediated mRNA decay (NMD) of aberrant P1-Runx1 
$m R N A$ is likely to be involved in reduction of P1-Runx 1 mRNA in Runx $1^{\text {P2TAG/P2TAG }}$ embryos. With these observations, we concluded that the phenocopying of Run $x 1^{\text {P2TAG/P2TAG }}$ and Run $x 1^{\Delta / \Delta}$ embryos was likely caused by a combined loss of the P1-Runx1 isoform and attenuated or loss of P2-Runx1 isoform expression.

\section{Characterization of Runx $1^{\text {P2TAA/P2TAA }}$ mutant mice}

Having shown that the replacement of ATG with TAG created an aberrant splice acceptor site, we designed a second target vector to replace the ATG with TAA, another stop codon, hereafter referred to as a $\operatorname{Run} \times 1^{\text {P2TAA }}$ mutation (Fig. 3a). Since we wanted to examine the effect of P2-Runx1 promoter deletion and the Runx $1^{\text {P2TAA }}$ mutation separately, we constructed two vectors, which would target the Runx $1^{\text {P2TAA }}$ mutation or P2-Runx1 promoter deletion (Fig. 3a), in the second gene targeting. We isolated ES clones harboring the Runx1 $1^{\text {P2TAA }}$ mutation (Fig. 3b), from which we established a mutant mouse line. However, although we isolated ES clones harboring the P2-Runx1 promoter deletion (Fig. 3c) and generated chimeric mice, the P2-Runx1 promoter deletion was not transmitted to the next generation. Therefore we could not generate the mouse line harboring the P2-Runx1 promoter deletion alone.

Analysis of liver cells from $11.5 \mathrm{dpc}$ embryos obtained from intercrossing Runx $1^{+/ P 2 T A A}$ heterozygotes detected $\mathrm{Lin}^{-} \mathrm{C}^{-\mathrm{Kit}^{+}} \mathrm{ScaI}^{+}$cells in the Run $x 1^{\text {P2TAA/P2TAA }}$ embryos
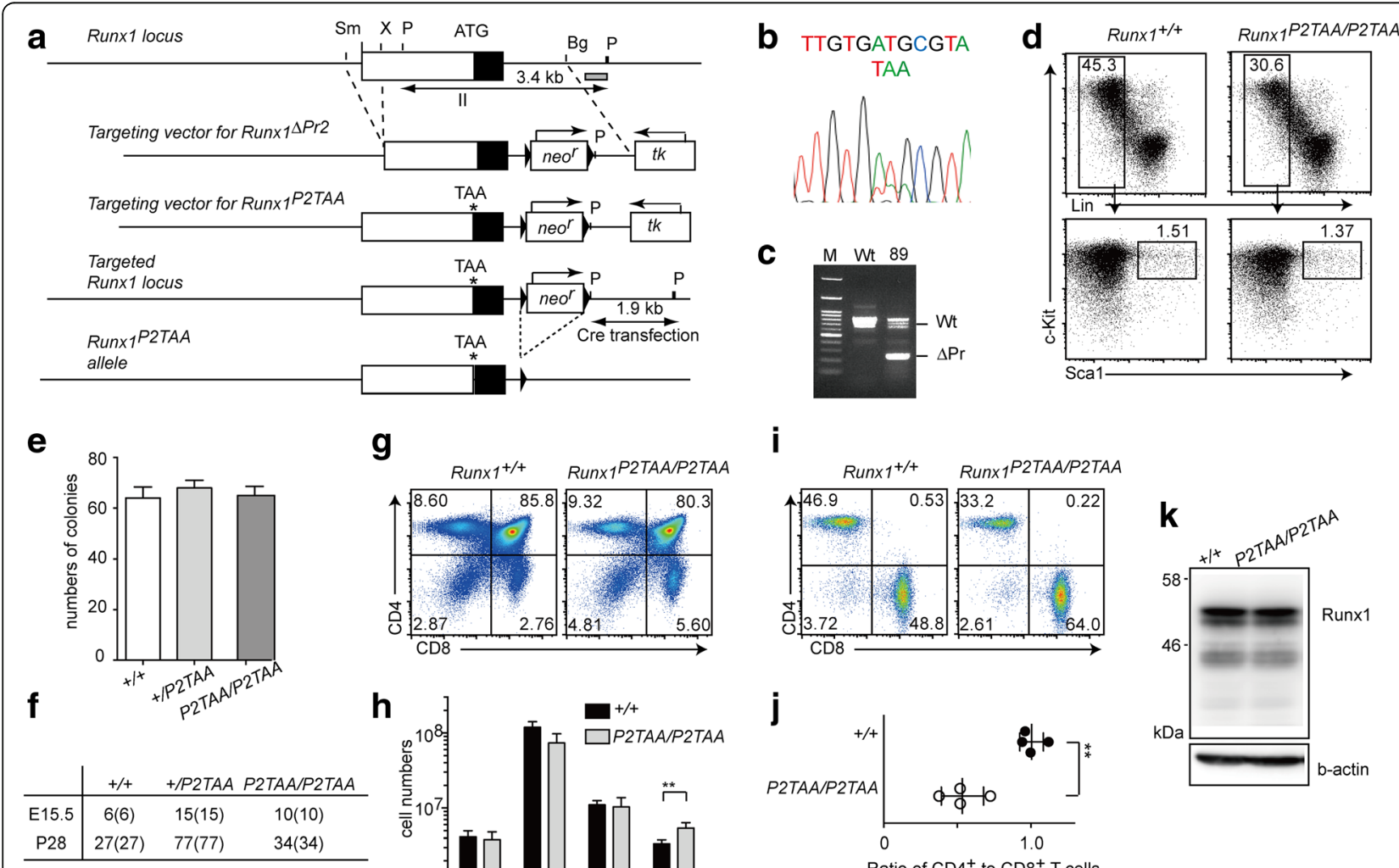

i
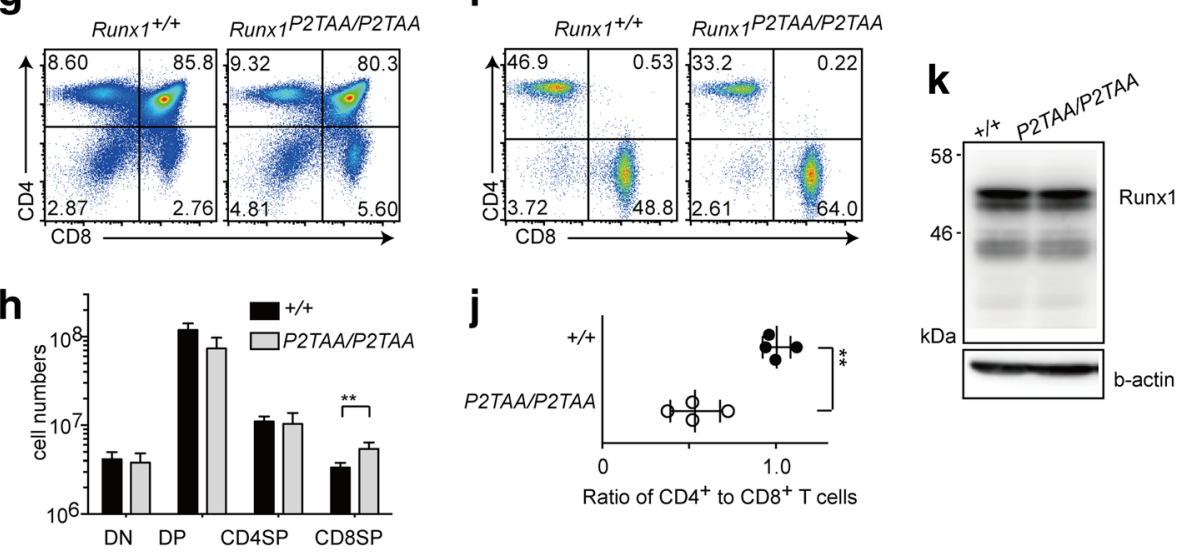

Fig. 3 Generation of Run $x 1^{\text {P2TAA }}$ mutant mice. a Schematic representation of the targeting strategy used to generate the Runx $1^{\text {P2TAA }}$ allele. A targeting vector was designed to replace ATG with TAA, marked with *. Open and closed boxes represent the $5^{\prime}$ untranslated region (UTR) and coding region in exon II, respectively. Neomycin resistance (neo') and thymidine kinase ( $t k$ ) genes were used for positive and negative selection, respectively. Triangles represent loxP sequences. b Sequence analysis of the genomic region around the ATG in exon II of the Runx1 gene in an ES clone showing replacement of ATG with TAA. c Gel image of DNA-PCR analysis showing incorporation of the P2-promoter deletion in an ES clone. $\mathbf{d}$ One representative dot plots of at least three individual experiments showing Scal and c-Kit expression in Lin-negative (Lin ${ }^{-}$) fetal liver cells from 11.5 dpc Run $x 1^{+/+}$and Run $x 1^{\text {P2TAAP2TAA }}$ embryos. e One representative of two colony forming assay of fetal liver cells from mice of indicated genotypes. f Genotyping of offspring obtained by intercrossing Run $x 1^{+/ P 2 T A A}$ heterozygous mice at $15.5 \mathrm{dpc}$ and four weeks (P28). Numbers and those in parenthesis represent live and total mice, respectively. $\mathbf{g}$ Dot plots showing CD4 and CD8 expression in total thymocytes of three week-old Runx $^{+/+}$and $R u n \times 1^{P 2 T A N}$ ${ }_{\text {P2TAA }}$ mice. $\mathbf{h}$ Graph showing absolute numbers of thymocyte subsets. DN: CD4 ${ }^{-} \mathrm{CD} 8^{-}$double negative. DP: $\mathrm{CD} 4^{+} \mathrm{CD} 8^{+}$double positive. CD4SP: $\mathrm{CD}^{+} \mathrm{CD}^{-}$single positive. CD8SP: $\mathrm{CD}^{-} \mathrm{CD}^{+}$single positive. Mean $\pm \mathrm{SD}$. ${ }^{*} P<0.01$. i Dot plots showing CD4 and CD8 expression in splenic T cells of

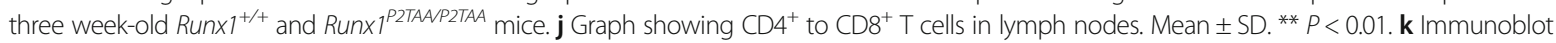
showing expression of Runx 1 protein in total thymocytes from three week-old Runx $\times 1^{+/+}$and RunX $1^{\text {P2TAAP2TAA }}$ mice. One representative image of two experiments 
to the same extent as in control embryos (Fig. 3d). Colony forming activity in fetal liver cells was also comparable between Run $x 1^{+/+}$and Run $x 1^{\text {P2TAA/P2TAA }}$ embryos (Fig. 3e). Thus, early definitive hematopoiesis during embryogenesis was not affected by the Runx $1^{P 2 T A A}$ mutation. In addition, we observed that homozygous Run $x 1^{\text {P2TAA/P2TAA }}$ mice were alive at birth and grew normally (Fig. 3f).

$\mathrm{T}$ lymphocyte development has been shown to be sensitive to Runx1 dosage. For example, the ratio of CD4-helper to CD8-cytotoxic $\mathrm{T}$ subsets in peripheral lymphoid tissues was shown to be reverted in heterozygous $R u n x 1^{+/ \Delta}$ mice [25]. We therefore examined T cell development in 3-5 week-old Runx $1^{\text {P2TAA/P2TAA }}$ mice. There were no significant changes in total thymocyte number, but the percentage and absolute number of CD8SP thymocyte subsets was slightly increased (Fig. $3 \mathrm{~g}$ and $\mathrm{h}$ ). The ratio of $\mathrm{CD}^{+}$to $\mathrm{CD}^{+} \mathrm{T}$ cells was also reverted in peripheral lymphoid tissues such as the spleen in Runx $1^{\text {P2TAA/P2TAA }}$ mice (Fig. 3i and j). This finding suggests that Runx1 activity is compromised in RUn $x 1^{\text {P2TAA/P2TAA }}$ mice. However, the total amount of Runx1 protein, as analyzed by immunoblotting, was similar between $R U n x 1^{+/+}$and Run $x 1^{\text {P2TAA/P2TAA }}$ thymocytes (Fig. 3k), presumably because the P1-Runx1 isoform is expressed in those cells.

\section{Generation of Runx $1^{\triangle P 1: P 2 T A A}$ Mouse line}

Previous reports have suggested the existence of truncated Runx2 and Runx3 proteins due to a non-canonical AUG codon downstream of the canonical AUG $(+1)[26,27]$.

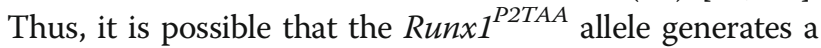
truncated Runx1 protein from the P2-Runx1 transcript via a non-canonical AUG codon, which would be produced in addition to the normal P1-Runx1 isoform. However, it was not possible to analyze whether and what types of truncated Runx1 protein were expressed from the Run $x 1^{P 2 T A A}$ allele, because there are no available antibodies that will distinguish between these two proteins. Theoretically, it is possible that a similar truncated P2Runx1 protein could be generated from the Run $x 1^{\text {P2TAG }}$ allele. However, such a putative truncated protein would not be sufficient to support early embryogenesis in the absence of the P1-Runx1 isoform. To overcome these limitations, and test whether elimination of the P1-Runx1 isoform over the Runxi $1^{P 2 T A A}$ allele recapitulates the Runx $1^{\text {P2TAG }}$ phenotype, we generated another mutant Run $x 1$ allele, referred to as $\operatorname{Run} x 1^{\triangle P 1: P 2 T A A}$, by targeting

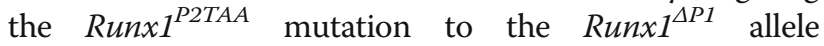
(Additional file 1).

Unexpectedly, homozygous Runx1 $1^{\text {DP1:P2TAA/ } \triangle P 1: P 2 T A A}$ mice were born alive and grew normally (Fig. 4a). Thus, not only is the RUnx $1^{\triangle P 1: P 2 T A A}$ allele completely different from the Runx1 $1^{\text {P2TAG }}$ allele, but the truncated P2-Runx1 protein generated from the $R u n x 1^{\triangle P 1: P 2 T A A}$ allele also provides sufficient Runx1 function for early mouse development. We then examined Runx1 protein expression in total thymocytes by immunoblotting. In $R u n x 1^{P I N / P I N}$ thymocytes that lack exon I of the RunxI gene, two different sizes of Runx1 protein were detected (Fig. 4b), indicating that the P2-Runx1 transcript produced two isoform protein. Interestingly, the protein with the heavier molecular weight was not detected in $R u n x 1^{\triangle P 1: P 2-}$ TAA/ $\triangle P 1: P 2 T A A$ cells (Fig. 4b). Given that the major difference between the $\operatorname{Run} x 1^{P 1 N}$ and $\operatorname{Run} x 1^{\triangle P 1: P 2 T A A}$ alleles is the ATG to TAA replacement in exon II, the P2-Runx1 isoform detected at the upper position in Run $x 1^{P I N / P 1 N}$ cells should be P2-Runx1 protein translated from canonical AUG $(+1)$, whereas the protein detected at the lower position was likely translated from the non-canonical downstream AUG. The truncated P2Runx1 protein detected in Runx $1^{\triangle P 1: P 2 T A A / \triangle P 1: P 2 T A A}$ cells was of a similar size to the one detected at the lower position in $\operatorname{Runx} 1^{P 1 N / P 1 N}$ cells, suggesting that both cells utilize the same non-canonical downstream AUG. There are AUGs at positions +73 and +151 , as well as others further downstream, which are in frame with the canonical AUG $(+1)$. Functional compensation by the truncated P2-Runx1 isoform disfavors the option to use AUG $(+151)$, because the truncated protein would lack the first two amino acids of the Runt-domain. In addition, the sequence around AUG (+73) was predicted to be closer to Kozak sequences than AUG $(+151)$ according to the ATGpr program [28]. We therefore propose that the position of the non-canonical downstream AUG is at +73 . Interestingly, we found that the amount of truncated P2-Runx1 isoform in $R u n x 1^{\triangle P 1: P 2-}$ TAA/ $\triangle P 1: P 2 T A A$ thymocytes was higher than that of Runx1 protein in $R u n x 1^{+/+}$and $R u n x 1^{P I N / P I N}$ cells (Fig. 4b). Semi-quantitative RT-PCR showed no clear differences in the amount of P2-Runxl transcript between the Run $x 1^{\text {PIN }}$ and Runx1 $1^{\triangle P 1: P 2 T A A}$ alleles (Fig. 4c), suggesting that post-transcriptional mechanisms may regulate the stability of the truncated P2-Runx1 protein. We then tested whether proteasome mediated degradation is involved in stability of truncated Runx1 protein by using proteasome inhibitor, MG132, and observed that amount both wild-type and truncated Runx1 proteins were slightly increased by treatment of $\mathrm{CD} 4^{+} \mathrm{T}$ cells with MG132 (Fig. 4d).

Next, we examined whether translation of Runx1 protein from the non-canonical AUG occurs in nonhematopoietic cells and from an artificial P2-Runx1 transcript derived from a plasmid, which has a short (10 bp) 5'UTR region. Three expression vectors were constructed by inserting a cDNA fragment encoding either wild-type P2-Runx1 or mutant cDNA fragments harboring a TAG or TAA replacement at the canonical ATG (+1) into a pcDNA3 expression vector. These were 

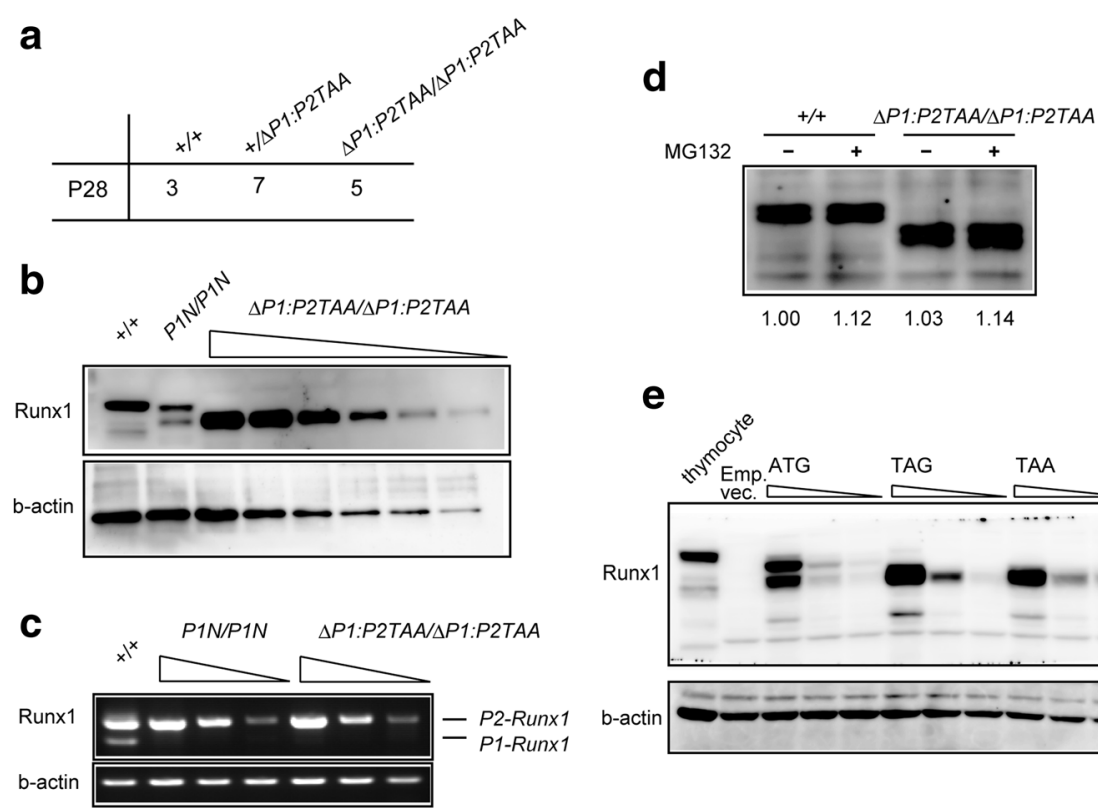

$\mathbf{e}$

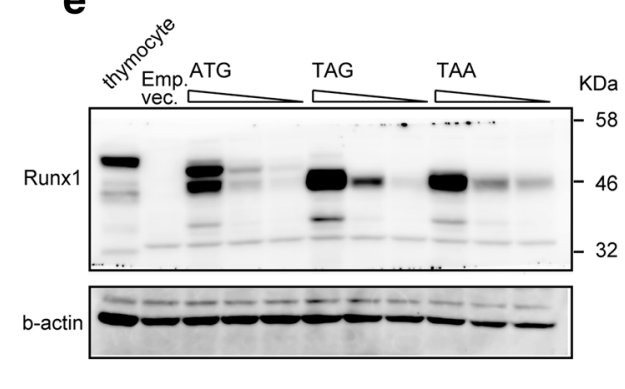

Fig. 4 Expression of truncated Runx1 protein from the Runx $1^{\triangle P 1: P 2 T A A}$ allele. a Genotyping of offspring obtained by intercrossing Runx $1^{\triangle P 1: P 2 T A A}$ heterozygous mice at four weeks. $\mathbf{b}$ Cell lysates prepared from total thymocytes of adult mice of indicated genotypes were immunoblotted with an anti-Runx 1 antibody. Serial dilutions, each reduced by half, were prepared from cell lysates from Run $1^{\triangle P 1: P 2 T A A V D \text { P1:P2TAA }}$ mice. The membrane was re-blotted with an anti b-actin antibody as an internal control. One representative image of two experiments. c Semi-quantitative RT-PCR analysis showing similar amounts of P2-Runx1 transcript between RunX1 ${ }^{\text {PIN/PIN }}$ and Runx $1^{\triangle P 1: P 2 T A A / \triangle P 1: P 2 T A A}$ thymocytes. $\mathbf{d}$ Effect of proteasome inhibitor, MG132, on the amount of Runx1 proteins. $C D 4^{+} T$ cells prepared from Runx $1^{+/+}$and Runx $1^{\triangle P 1: P 2 T A A / \triangle P 1: P 2 T A A}$ mice were treated with $10 \mu M$ MG132 proteasome inhibitor for one hour. Numbers at the bottom indicate relative expression level to that of Runx $1^{+/+}$cells without MG132 treatment. e Expression of Runx1 protein from three expression vectors harboring different sequences around the translation start site, ATG, TAG and TAA, on P2-Runx1 cDNA. Different amounts (1.0, 0.5, and $0.25 \mu \mathrm{g})$ of these vectors were transfected into $293 \mathrm{~T}$ cells. Forty-eight hours after transfection, cell lysates were immunoblotted with an anti-Runx1 antibody. Empty vector (Emp.Vec.) and thymocyte lysates were included as references

then transfected into non-hematopoietic 293 T cells. As observed in thymocytes, two Runx1 isoforms were produced from the wild-type P2-Runx1 construct, while both the TAG and TAA mutant constructs generated only the smaller Runx1 isoform (Fig. 4e). These results suggest that use of the non-canonical AUG is not specific to thymocytes, and that it is not affected by the TAG mutation.

\section{Restored basophil development in Runx $1^{\triangle P 1 / P 2 T A A}$ mice}

Only the truncated Runx1 protein lacking unique Nterminal sequences specific to either P1-Runx1 or P2Runx1 were expressed in Runx1 $1^{\triangle P 1: P 2 T A A /} \triangle P 1: P 2 T A A$ mice, but we did not observe any major defects in early hematopoiesis or thymocyte development (Fig. 5a). However, the reverted CD4/CD8 ratio observed in peripheral lymphoid tissues of $R u n x 1^{P I N / P I N}$ mice was restored to some extent in the $R u n x 1^{\triangle P 1: P 2-}$ TAA/ $\triangle P 1: P 2 T A A$ mice, although there was still some skewing towards the CD8-lineage (Fig. 5a and b). These observations indicate that the increased amount of truncated P2-Runx1 in Runx1 $1^{\triangle P 1: P 2 T A A /} \triangle P 1: P 2 T A A$ cells compared to Runx $1^{\text {PIN/PIN }}$ cells has the potential to restore the defect in hematopoietic cell differentiation caused by loss of the P1-Runx1 isoform. Since we had previously observed that basophil development was impaired in $R u n x 1^{P 1 N / P 1 N}$ mice [19], we next examined whether basophil development remained impaired or was restored in $R u n x 1^{\triangle P 1: P 2 T A A /}$ $\triangle P 1: P 2 T A A$ mice. Basophils can be detected in the bone marrow as a $\mathrm{CD}_{4} 9 \mathrm{~b}^{+} \mathrm{IgE}^{+}$or $\mathrm{Fc}_{\mathrm{R}} \mathrm{RI}^{+} \mathrm{IgE}^{+}$population. As previously reported, these cell populations were reduced in Runx $1^{P 1 N / P 1 N}$ mice (Fig. $5 \mathrm{c}$ and d). On the contrary, the percentage and number of basophils in

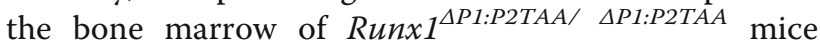
were restored to levels comparable to control mice (Fig. $5 \mathrm{c}$ and d). Furthermore, levels of Mcpt8 gene expression, a molecular marker of basophils, were also restored in the bone marrow cells of $\operatorname{Run} \times 1^{\triangle P 1: P 2 T A A}$ / $\triangle P 1: P 2 T A A$ mice (Fig. 5e). This provides further support that the truncated P2-Runx1 isoform derived from the Run $x 1^{\triangle P 1: P 2 T A A}$ allele, compared to total P2-Runx1 protein that consists of both wild-type and truncated P2-Runx1 protein from the Runx $1^{P 1 N}$ allele, can compensate for P1-Runx1 function more efficiently during basophil development. 
a Total
thymocytes

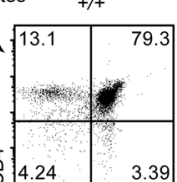

P1N/P1N $\triangle P 1: P 2 T A A / A P 1: P 2 T A A$

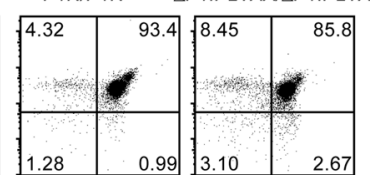

$\stackrel{\mathrm{LN}}{\mathrm{TCR} \beta^{+}}$

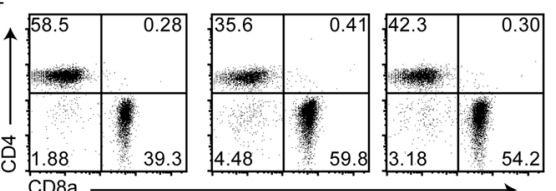

C

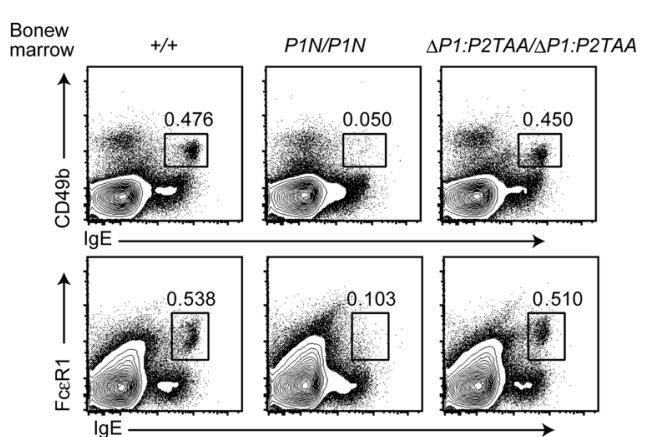

b

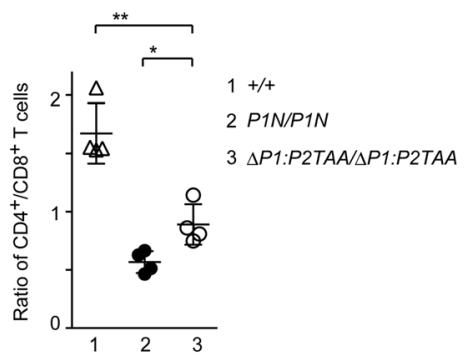

d

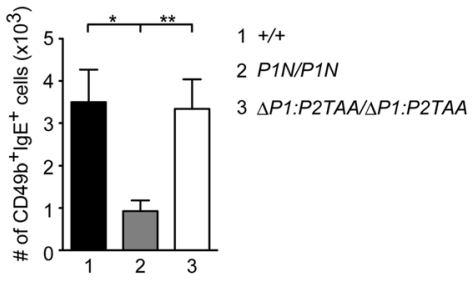

e

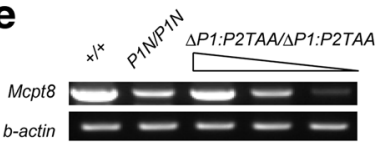

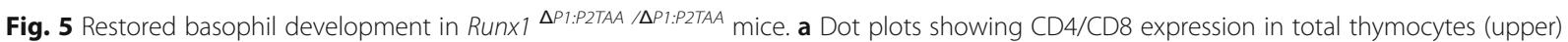
and lymph node T cells (lower) of 4 to 6 week-old RunX1 $1^{\text {PIN/P1N }}$ and Runx $1^{\triangle P 1: P 2 T A A: \triangle P 1: P 2 T A A}$ mice. $\mathbf{b}$ Graph showing CD4 ${ }^{+}$T cells to CD8 $^{+}$T cells

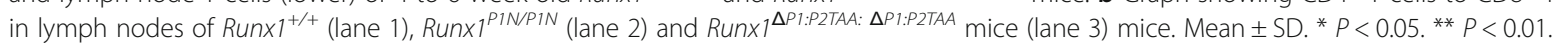
c Flow cytometry analysis of basophil differentiation. Dot plots showing expression of IgE, CD49b and FcERI in bone marrow cells from Runx $1^{+/+}$,

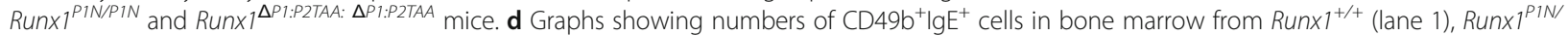
${ }^{P I N}$ (lane 2) and Runx1 ${ }^{\triangle P 1: P 2 T A A: \triangle P 1: P 2 T A A}$ mice (lane 3) mice. Mean \pm SD. ${ }^{*} P<0.05, * * P<0.01$. e Semi-quantitative RT-PCR analysis of McPt8 transcript, a molecular marker of basophils, in bone marrow cells from mice of indicated genotypes. One representative of two experiments

\section{Discussion}

In the present study, we generated three novel Runx 1 mutant mouse models, Runx $1^{\text {P2TAA/P2TAA }}$, Run $x 1^{\text {P2TAG/ }}$ P2TAG and RUnX1 $1^{\triangle P 1: P 2 T A A /} \triangle P 1: P 2 T A A$, and addressed the function of the P2-Runx1 transcript during hematopoiesis. Unexpectedly, a mutation that replaced the canonical translational AUG (+1) codon with UAG, named the Runx $1^{P 2 T A G}$ allele, created an aberrant splice acceptor site. Joining of the P1-Runx1 transcript using this aberrant splice acceptor not only occurred more efficiently than that from the canonical splice acceptor, but also resulted in a frame shift. The P1Runx1 transcript from the Runx $1^{\text {P2TAG }}$ allele thus could generate mostly short peptides that lacked the Runt-domain. The mechanism by which splice donor/ acceptor sites are selected and utilized remains unclear, therefore it is difficult to identify the reason for preferential use of the aberrant splice acceptor created by the TAG replacement. In the Runx $1^{P 2 T A G}$ allele, we deleted a 580 bp genomic region that included a $200 \mathrm{bp}$ region upstream of the transcriptional start site (TSS), which corresponds to a putative $P 2-R u n x 1$ promoter region. However, we still observed a P2-Runx1 transcript in Runx $1^{\text {P2TAG/P2TAG }}$ embryos at $11.5 \mathrm{dpc}$, suggesting the presence of another promoter(s) that drives transcription from a site close to exon II. Indeed, a recent FANTOM5 database comprising numerous TSS from Cap Analysis of Gene Expression (CAGE) identified a cryptic TSS that mapped to a region $220 \mathrm{bp}$ downstream of the canonical TSS for the P2-Runx1 transcript (Additional file 2). Although this second TSS was also deleted in the Runx $1^{\text {P2TAG }}$ allele, it raises the possibly that additional promoter(s) could drive P2-Runx1 transcription. It has been proposed that shadow enhancers become active when the primary enhancer becomes non-functional, in order to ensure robustness [29]. Thus, a similar backup system may be in place to activate a hidden promoter, thus ensuring transcription of P2-Runx1 from the Runx $1^{P 2 T A G}$ allele.

Production of the same truncated Runx1 protein from expression vectors harboring either a TAG or TAA replacement suggests that a truncated Runx1 
protein may be produced from the P2-Runx 1 transcript transcribed from the Runx1 $1^{\text {P2TAG }}$ allele. However, in contrast to $R U n x 1^{\triangle P 1: P 2 T A A / \triangle P 1: P 2 T A A}$ mice, which survive beyond birth, Runx $1^{\text {P2TAG/P2TAG }}$ embryos die at $12.5 \mathrm{dpc}$, as is also seen in $R u n x 1^{\Delta / \Delta}$ embryos. Therefore, even though a truncated P2-Runx1 isoform is produced from the P2-Runx1 transcript driven by a putative shadow promoter in $\operatorname{Run} x 1^{\text {P2TAG/P2TAG }}$ mice, the amount is unlikely to be sufficient to support mouse embryogenesis. This is supported by the previous observation that embryos expressing a compound mutation of the Runx $1^{\text {P2Neo }}$ with the $\operatorname{Run} x 1^{P 1 N}$ allele (Runx1 ${ }^{P 2 N e o / P 1 N}$ embryos) also died at $12.5 \mathrm{dpc}$. Based on the earlier expression of the P2-Runx1 transcript and the presence of conserved functional Runx sites within the P1-Runx1 promoter $[13,22]$, it has been proposed that the P2-Runx1 isoform may activate the P1-Runx1 promoter. It was also shown that mice lacking the IRES sequences in the P2-RunxI transcript died around $14.5 \mathrm{dpc}$ [30]. Thus, expression of P2Runx1 above a certain amount is necessary to support mouse embryogenesis until the P1-Runx 1 promoter is fully activated and able to produce enough P1-Runx1 isoform to compensate for low levels of the P2-Runx1 isoform.

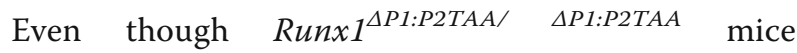
produced only the truncated P2-Runx1 protein, whose translation would start from the AUG $(+73)$, these mice grew normally and did not display any major defects in hematopoiesis. These findings indicate that $\mathrm{N}$-terminal sequences specific to either the P1-Runx1 or P2-Runx1 isoform are dispensable for most Runx1 functions. Rather, an increase in the amount of truncated P2-Runx1 protein, which is presumably regulated at the post-transcriptional level, suggests that the Nterminal amino acid sequences (Arg2 to Lys24) that are lost in the truncated P2-Runx1 isoform function as a negative regulatory domain for protein stability. A previous study showed that the $\mathrm{N}$-terminal region of the human Runx1 protein contains an auto-inhibitory domain that inhibits hetero-dimerization with $\mathrm{Cbf} \beta /$ PEBP2 $\beta$ [31]. Dimerization with Cbf $\beta /$ PEBP2 $\beta$ prevents ubiquitin-mediated degradation and is therefore important for stability of the Runx1 protein [32]. The truncated Runx1 protein that is translated from the non-canonical AUG $(+73)$ lacks a lysine residue (Lys24), and it is possible that this Lys24 serves as an ubiquitination site, since its replacement by arginine (Lys24Arg) was shown to increase protein stability [32]. Thus, escaping protein degradation, through either enhanced dimerization or reduced ubiquitination due to lack of the $\mathrm{N}$-terminal, is at least partly responsible for the increase in truncated P2-Runx1 protein. In this case, the fact that more of the canonical P2-
Runx1 isoform than the truncated P2-Runx1 isoform is produced in thymocytes from $R u n x 1^{P 1 N / P 1 N}$ mice suggests that the efficacy of translation is higher from the canonical AUG (+1).

Finally, our results revealed that the truncated Runx1 protein produced from the $R u n x 1^{\triangle P 1: P 2 T A A}$ allele was able to restore the defect in basophil development due to absence of the P1-Runx1 isoform. The enhanced function of the truncated P2-Runx1 isoform over the canonical P2-Runx1 isoform is likely to stem mainly from the higher amount, although other possibilities, such as changes in affinity for partner proteins due to conformational changes, cannot be formally excluded. On the other hand, the reverted CD4/CD8 $\mathrm{T}$ cell ratio was not completely restored by the truncated P2Runx1 isoform. A previous study showed that overexpression of the P2-Runx1 isoform from a transgene caused a skew towards CD8-lineage differentiation [33]. Along with a CD8-skewing in the presence of P1Runx1 in the Runx $1^{\text {P2TAA/P2TAA }}$ mice, an increase in the amount of truncated P2-Runx1 could have a similar effect on $\mathrm{T}$ lymphocyte development. Given the lack of P2-Runx1-specific N-terminal sequences in the truncated protein, this effect would not be specific to the canonical P2-Runx1 isoform. A similar CD8-skew in differentiation was observed with a half-dosage of the Runx1 gene [25], suggesting that tight regulation of the amount of Runx1 protein is essential for appropriate $\mathrm{T}$ lymphocyte development. It is interesting that the canonical AUG $(+1)$ is predominately used to generate the canonical P2-Runx1 isoform, which is more unstable than the truncated P2-Runx1 isoform. Together with evolutionary conservation of the $\mathrm{N}$ terminal sequences in P2-Runx1 proteins, any potential negative regulatory function endowed on the $\mathrm{N}$-terminal sequences of the Runx1 protein could have a physiological role in adjusting the amount of Runx1 protein to appropriate levels.

\section{Conclusions}

Generation of isoform proteins by differential usage of alternative promoter or RNA splicing contributes to increase functional diversification of the gene product. The findings by our genetic approaches modulating the translation start codon on the P2-Runx2 transcript, which were combined with loss of the P1-Runx1 isoform, unraveled not only that unique $\mathrm{N}$-terminal sequences specific to P1-Runx1 or P2-Runx2 are dispensable for Runx1 function supporting embryogenesis and early hematopoiesis, but also that the $\mathrm{N}$-terminal sequences in the P2-Runx1 isoform have a role in finetuning the Runx1 protein level through de-stabilizing P2-Runx1 isoform. 


\section{Additional files}

Additional file 1: Strategy used to generate Runx1DP1:P2TAA mutant allele by sequential gene targeting. Schematic representation of the targeting strategy used to generate the Runx1DP1:P2TAA allele. Open and closed boxes represent the $5^{\prime}$ untranslated region (UTR) and coding region in exon I and II, respectively. The neor and tk indicate neomycin resistance and thymidine kinase genes, respectively. Triangles represent loxP sequences. To select ES cells with G418 after transfection of the target vector for the Run $x 1^{\text {P2TAA }}$ mutation, the neor gene was removed from ES clones harboring the RunX1+/PIN genotype, thus generating ES clones harboring the Runx $1^{+/ \Delta P 1}$ genotype. Cells were transfected with the target vector for the Runx $1^{\text {P2TAA }}$ mutation and clones that underwent homologous recombination were isolated. To screen for whether the Runx1 or Runx1DP1 allele was targeted to the Runx $1^{\text {P2TAA }}$ mutation, ES clones were transduced with a retroviral vector encoding Cre recombinase and screened by PCR for an inverted recombination event between loxP sequences in opposite directions. ES clones harboring the Run $\times 1^{+/ \triangle P 1: P 2 T A A N}$ genotype were isolated. Primers are indicated as red arrowheads. Gel image on the right shows detection of inverted recombination in clones 3-8 and 9-8. The neor gene was removed by transient transfection of Cre recombinase to isolate ES clones harboring the $R$ Run $x 1^{+/ \triangle P 1: P 2 T A A}$ genotype. (PDF $209 \mathrm{~kb}$ )

Additional file 2: Detection of a cryptic TSS that mapped to a region 220 bp downstream of the canonical TSS in the public FANTOM5 database. Image of FANTOM5 web browser showing canonical and cryptic transcriptional start site (TSS), which are marked with arrow heads, for P2-Runx1 transcript. Red line indicates a genomic region that was deleted in the Runx1P2TAG allele. Numbers represent nucleotide positons according to $\mathrm{mm} 9$ reference. (PDF $84 \mathrm{~kb}$ )

\section{Abbreviations}

AML: Acute myeloid leukemia; CAGE: Cap Analysis of Gene Expression; dpc: Days post coitum; ES cells: Embryonic stem cells; FceRl: Fc epsilon receptor I; HSC: Hematopoietic stem cell; Mcpt8: Mast cell protease 8; RTPCR: Reverse transcription-polymerase chain reaction; Runx: Runt-related transcription factors; TSS: Transcriptional start site

\section{Acknowledgments}

We thank S. Nishimoto and M. Tenno for vector construction, and T. Ishikura for ES cell aggregation.

\section{Funding}

This work was supported by JSPS KAKENHI Grant Number JP 21229008 (I.T.) JP 26293109 (I.T.) and JP 22021045 (I.T)

\section{Availability of data and materials}

The datasets and materials used and/or analyzed during the current study are presented in the main paper and additional files.

\section{Authors' contributions}

SN, NY, KK, and TY performed phenotypic analyses of mice. S.M. isolated ES clones. I.T. designed experiments and wrote the manuscript. All authors read and approved the final manuscript.

\section{Ethics approval}

Animal work was performed in accordance with institutional guidelines and RIKEN Yokohama Campus regulations and was authorized by the Safety Department at RIKEN Yokohama Campus (28-017).

\section{Consent for publication}

Not applicable

\section{Competing interests}

The authors declare that they have no competing interests.

\section{Publisher's Note}

Springer Nature remains neutral with regard to jurisdictional claims in published maps and institutional affiliations.

\section{Author details}

'Laboratory for Transcriptional Regulation, RIKEN Center for Integrative Medical Sciences (IMS). 1-7-22 Suehiro-cho, Tsurumi-ku, Yokohama 230-0045, Japan. ${ }^{2}$ Abteilung Immunologie, Interfakultaeres Institute fuer Zellbiologie, Auf der Morgenstelle 15, 72076 Tuebingen, Germany. ${ }^{3}$ Laboratory for Immune Crosstalk, RIKEN Center for Integrative Medical Sciences (IMS), 1-7-22 Suehiro-cho, Tsurumi-ku, Yokohama 230-0045, Japan. ${ }^{4}$ Cancer Science Institute of Singapore, National University of Singapore, 14 Medical Drive, \#12-01, Singapore 117599, Singapore. ${ }^{5}$ Faculty of Life Sciences (Microbiology), University of Central Punjab, 1 - Khayaban-e-Jinnah Road, Johar Town, Pakistan. ${ }^{6}$ International Research Center for Medical Sciences, Kumamoto University, 2-2-1 Honjo, Chuo-ku, Kumamoto City 860-0811, Japan.

Received: 7 July 2017 Accepted: 1 October 2017

Published online: 18 October 2017

\section{References}

1. Ito Y, Bae SC, Chuang LS. The RUNX family: developmental regulators in cancer. Nat Rev Cancer. 2015;15(2):81-95.

2. de Bruijn MF, Speck NA. Core-binding factors in hematopoiesis and immune function. Oncogene. 2004;23(24):4238-48.

3. Levanon D, Groner Y. Structure and regulated expression of mammalian RUNX genes. Oncogene. 2004;23(24):4211-9.

4. Coffman JA. Is Runx a linchpin for developmental signaling in metazoans? J Cell Biochem. 2009;107(2):194-202.

5. Nimmo R, Woollard A. Worming out the biology of Runx. Dev Biol. 2008: 313(2):492-500

6. Okuda T, van Deursen J, Hiebert SW, Grosveld G, Downing JR. AML1, the target of multiple chromosomal translocations in human leukemia, is essential for normal fetal liver hematopoiesis. Cell. 1996;84(2):321-30.

7. Wang $Q$, Stacy $T$, Binder M, Marin-Padilla M, Sharpe AH, Speck NA. Disruption of the Cbfa2 gene causes necrosis and hemorrhaging in the central nervous system and blocks definitive hematopoiesis. Proc Nat Acad Sci U S A. 1996;93(8):3444-9.

8. Engel ME, Hiebert SW. Proleukemic RUNX1 and CBFbeta mutations in the pathogenesis of acute leukemia. Can Treat Res. 2010;145:127-47.

9. Mikhail FM, Sinha KK, Saunthararajah Y, Nucifora G. Normal and transforming functions of RUNX1: a perspective. J Cell Physiol. 2006;207(3): 582-93.

10. Osato M. Point mutations in the RUNX1/AML1 gene: another actor in RUNX leukemia. Oncogene. 2004:23(24):4284-96.

11. Ghozi MC, Bernstein $Y$, Negreanu V, Levanon D, Groner $Y$. Expression of the human acute myeloid leukemia gene AML1 is regulated by two promoter regions. Proc Nat Acad Sci U S A. 1996:93(5):1935-40.

12. Pozner A, Goldenberg D, Negreanu V, Le SY, Elroy-Stein O, Levanon D, Groner Y. Transcription-coupled translation control of AML1/RUNX1 is mediated by cap- and internal ribosome entry site-dependent mechanisms. Mol Cell Biol. 2000:20(7):2297-307.

13. Telfer JC, Rothenberg EV. Expression and function of a stem cell promoter for the murine CBFalpha2 gene: distinct roles and regulation in natural killer and T cell development. Dev Biol. 2001;229(2):363-82.

14. Levanon D, Bernstein Y, Negreanu V, Ghozi MC, Bar-Am I, Aloya R, Goldenberg D, Lotem J, Groner Y. A large variety of alternatively spliced and differentially expressed mRNAs are encoded by the human acute myeloid leukemia gene AML1. DNA Cell Biology. 1996;15(3):175-85.

15. Bee T, Liddiard K, Swiers G, Bickley SR, Vink CS, Jarratt A, Hughes JR, Medvinsky A, de Bruijn MF. Alternative Runx1 promoter usage in mouse developmental hematopoiesis. Blood Cells Mol Dis. 2009;43(1):35-42.

16. Bee T, Swiers G, Muroi S, Pozner A, Nottingham W, Santos AC, Li PS, Taniuchi I, de Bruijn MF. Nonredundant roles for Runx1 alternative promoters reflect their activity at discrete stages of developmental hematopoiesis. Blood. 2010;115(15):3042-50.

17. Sroczynska P, Lancrin C, Kouskoff V, Lacaud G. The differential activities of Runx1 promoters define milestones during embryonic hematopoiesis. Blood. 2009;114(26):5279-89.

18. Tachibana M, Tenno M, Tezuka C, Sugiyama M, Yoshida H, Taniuchi I. Runx1/Cbfbeta2 complexes are required for lymphoid tissue inducer cell differentiation at two developmental stages. J Immunol. 2011;186(3):1450-7.

19. Mukai K, BenBarak MJ, Tachibana M, Nishida K, Karasuyama H, Taniuchi I, Galli SJ. Critical role of P1-Runx1 in mouse basophil development. Blood. 2012;120(1):76-85 
20. Pozner A, Lotem J, Xiao C, Goldenberg D, Brenner O, Negreanu V, Levanon D, Groner Y. Developmentally regulated promoter-switch transcriptionally controls Runx1 function during embryonic hematopoiesis. BMC Dev Biol. 2007;7:84.

21. Fujita $Y$, Nishimura $M$, Taniwaki $M$, Abe $T$, Okuda $T$. Identification of an alternatively spliced form of the mouse AML1/RUNX1 gene transcript AML1C and its expression in early hematopoietic development. Biochem Biophys Res Commun. 2001;281(5):1248-55.

22. Spender LC, Whiteman HJ, Karstegl CE, Farrell PJ. Transcriptional crossregulation of RUNX1 by RUNX3 in human B cells. Oncogene. 2005;24(11):1873-81.

23. Yokomizo T, Yamada-Inagawa T, Yzaguirre AD, Chen MJ, Speck NA, Dzierzak E. Whole-mount three-dimensional imaging of internally localized immunostained cells within mouse embryos. Nat Protoc. 2012;7(3):421-31.

24. Egawa T, Tillman RE, Naoe $Y$, Taniuchi I, Littman DR. The role of the Runx transcription factors in thymocyte differentiation and in homeostasis of naive T cells. J Exp Med. 2007;204:1945-57.

25. Hayashi K, Natsume W, Watanabe T, Abe N, Iwai N, Okada H, Ito Y, Asano M, Iwakura $Y$, Habu S, et al. Diminution of the AML1 transcription factor function causes differential effects on the fates of CD4 and CD8 singlepositive T cells. J Immunol. 2000;165(12):6816-24.

26. Kim B, Sasaki Y, Egawa T. Restriction of Nonpermissive RUNX3 Protein Expression in T Lymphocytes by the Kozak Sequence. J Immunol. 2015; 195(4):1517-23.

27. Okura H, Sato S, Kishikawa S, Kaneto S, Nakashima T, Yoshida N, Takayanagi $\mathrm{H}$, Kiyono $\mathrm{H}$. Runx2-I isoform contributes to fetal bone formation even in the absence of specific N-terminal amino acids. PLoS One. 2014;9(9): e108294.

28. Nishikawa T, Ota T, Isogai T. Prediction whether a human cDNA sequence contains initiation codon by combining statistical information and similarity with protein sequences. Bioinformatics. 2000;16(11):960-7.

29. Hong JW, Hendrix DA, Levine MS. Shadow enhancers as a source of evolutionary novelty. Science. 2008;321(5894):1314

30. Nagamachi A, Htun PW, Ma F, Miyazaki K, Yamasaki N, Kanno M, Inaba T, Honda Z, Okuda T, Oda H, et al. A 5' untranslated region containing the IRES element in the Runx1 gene is required for angiogenesis, hematopoiesis and leukemogenesis in a knock-in mouse model. Dev Biol. 2010;345(2):226-36.

31. Kim WY, Sieweke M, Ogawa E, Wee HJ, Englmeier U, Graf T, Ito Y. Mutual activation of Ets-1 and AML1 DNA binding by direct interaction of their autoinhibitory domains. EMBO J. 1999;18(6):1609-20.

32. Huang G, Shigesada K, Ito K, Wee HJ, Yokomizo T, Ito Y. Dimerization with PEBP2beta protects RUNX1/AML1 from ubiquitin-proteasome-mediated degradation. EMBO J. 2001;20(4):723-33.

33. Hayashi K, Abe N, Watanabe T, Obinata M, Ito M, Sato T, Habu S, Satake M. Overexpression of AML1 transcription factor drives thymocytes into the CD8 single-positive lineage. J Immunol. 2001;167(9):4957-65.

\section{Submit your next manuscript to BioMed Central and we will help you at every step:}

- We accept pre-submission inquiries

- Our selector tool helps you to find the most relevant journal

- We provide round the clock customer support

- Convenient online submission

- Thorough peer review

- Inclusion in PubMed and all major indexing services

- Maximum visibility for your research

Submit your manuscript at www.biomedcentral.com/submit 